\title{
Distribution and habitat attributes associated with the Himalayan red panda in the westernmost distribution range, Nepal
}

\author{
Saroj Shrestha ${ }^{1}$, Arjun Thapa ${ }^{2}$, Damber Bista ${ }^{3}$, Natasha Robinson $^{4}$, Ang Sherpa ${ }^{1}$, Krishna \\ Acharya $^{5}$, Shant Jnawali ${ }^{6}$, and Sony Lama ${ }^{1}$ \\ ${ }^{1}$ Red Panda Network \\ ${ }^{2}$ Small Mammals Conservation and Research Foundation \\ ${ }^{3}$ The University of Queensland \\ ${ }^{4}$ Australian National University \\ ${ }^{5}$ Ministry of Forests and Environment \\ ${ }^{6}$ WWF Nepal
}

September 11, 2020

\begin{abstract}
The Himalayan red panda (Ailurus fulgens) is of special interest in evolutionary studies due to its taxonomic uniqueness. Globally, Nepal represents the Westernmost edge of the red panda distribution. Fewer studies of red panda have been carried out which hinders the implementation of effective conservation actions of the species. We aim to determine important habitat features influencing the distribution of red panda and recommend possible habitat corridors. We conducted an extensive field survey and analyzed red panda presence data, key food resources (bamboo), and bioclimatic variables to build a Maxent habitat model and determine habitat requirements of the red panda. Himalayan red pandas were confined between the range of $2600 \mathrm{~m}-$ 3,600 m, with most records between $3250 \mathrm{~m}-3400 \mathrm{~m}$ on the north and west-facing slopes. The potentially suitable habitat of the red panda in Western Nepal is estimated to be about $3,222 \mathrm{~km} 2$ with a relative abundance of 3.34 signs $/ \mathrm{km}$. Important habitat attributes for red panda occurrence include aspects, canopy cover, bamboo cover, and distance to water. Combining species habitat requirements and disturbance factors (human footprint), we suggested five potential biological corridors in Western Nepal. Western Nepal has suitable habitat attributes for the red panda distributions. Ecological variables such as canopy cover, bamboo cover, distance to the water, and aspects are important attributes for red panda occurrence in Western Nepal alike Central Nepal. We suggested five potential corridors in Western Nepal; however, due to a lack of detailed knowledge on corridors and connectivity, a comprehensive field-based assessment is required to validate it scientifically. Keywords: Biological Corridor; Distribution; Habitat requirements; Red panda
\end{abstract}

\section{Introduction}

Information on species distribution and habitat use is one of the important aspects of wildlife ecology. Such information is critical for the successful conservation of the species (Braun 2005). Species distribution models are being widely used in wildlife studies to answer some of those key biological questions (Sinclair et al. 2006). Distribution models establish a quantitative relationship between the relative occurrence of species and their bio-physical and environmental conditions in the landscape (Guisan \& Zimmermann 2000; Elith et al. 2006; Phillips et al. 2004, 2006; Phillips \& Dudik 2008). These models can provide essential information on habitat suitability, and key habitat attributes affecting species' distribution. Such information obtained from the 
species distribution modelling eventually help identify and prioritize key conservation areas (Wilson et al. 2009).

Despite its broad geographical range across the Himalayas, red panda is patchily distributed and occurs at low densities (Thapa et al. 2018; Wei et al. 1999; Yonzon \& Hunter1991). The red panda has been recorded within the altitudinal range of 1,500 - 1,800 $\mathrm{m}$ (Choudhury 2001), with a resemblance to that of habitats with dense, undergrowth bamboo (Choudhury 2001; 2006; Zhang et al. 2006). Red pandas are charismatic animals that make them an ideal flagship species for harnessing public support for biodiversity conservation (Yonzon \& Hunter 1991a; Dorji et al. in press). However, this endangered species is vulnerable to extinction due to habitat loss and fragmentation (Yibo et al. 2020; Acharya et al. 2018; Glatston et al. 2015; Hu 2004; Williams 2003; Pradhan 2001; Choudhury 2001; Wei et al. 1999a and Glatston 1994). The poaching and demand of hides have further aggravated the threat in Nepal than other range countries (Bista et al. 2020; Badola et al. 2020). A new study classified red panda into two distinct species: Himalayan red panda (Ailurus fulgens ) and Chinese red panda (Ailurus styani), Hu et al. 2020. Of these, the former species inhabits in Nepal and is more vulnerable to threats compared to its Chinese relative (Yibo et al. 2020).

Red panda is a habitat and diet specialist species. Available studies suggest that bamboo cover, tree canopy cover, and proximity to water are important attributes (Bista et al. 2017; Bista et al. 2019; Thapa et al. 2020). Bamboo contribute more than $83 \%$ of the total red panda diet (Yonzon \& Hunter 1991b; Reid et al. 1991; Wei et al. 1999b). Being primarily a bamboo eater, the red panda has a very low metabolic rate (Wei et al. 2000). Red panda spends most of the time on foraging and sleeping on tree branches or in tree hollows during the day (Yonzon \& Hunter 1991a; Wei \& Zhang 2011). This animal use elevated objects, such as shrub branches, fallen logs, or tree stumps to reach bamboo leaves. This elusive animal frequently uses few latrine sites for defecation (Yonzon 1989). In Nepal, red panda presence has been documented from 24 districts and seven protected areas with potential habitat of $13,800 \mathrm{~km}^{2}$ to $24,000 \mathrm{~km}^{2}$ (Bista et al. 2016; Panthi et al. 2019; Thapa et al. 2020).

Similarly, occurring in a remote part of the Himalayan landscape, the red panda species remains poorly known, and the available database of the total population of species is possibly underestimated due to scarce records of occurrence. To date, most studies have focused on observational surveys of indirect indicators like feces and pugmarks (Pradhan et al. 2001; Wei, Feng, Wang, \& Hu, 1999; Yonzon \& Hunter, 1991) as well as discussions with specialists and local communities (Jnawali et al. 2012; Wei et al. 2014). A comprehensive study on distribution and habitat attributes in the western range is still lacking. Potential distribution and habitat-related attributes associated with red panda distribution has been broadly examined in Nepal (Thapa et al. 2019; Bista et al. 2017; Bista et al. 2019; Williams 2003). However, very few studies have discussed on red panda distribution and habitat use in the western range (Thapa 2019; Bhatta et al. 2014; Bista et al. 2019). Available studies have either covered western range in a small-scale (Bhatta et al. 2014), or covered it as a part of the large-scale study (Thapa et al. 2019; Panthi et al. 2019; Bista et al. 2019). Long-term conservation of red panda requires site-specific detailed information from this range. In addition, the habitat use trend vary across the distribution range due to uneven micro-habitat conditions (Bista et al. 2019). However, the available studies do not provide sufficient information on habitat use from the western-most distribution range. These facts underpin the need of a comprehensive study in this one of the least explored red panda range.

With this background, our study aims to build understanding on distribution and habitat requirements of Himalayan red panda from its western distribution range. We intend to (a) document red panda distribution, (b) estimate abundance, and (c) identify habitat variables affecting distribution in the western Nepal. 


\section{Material and Methods}

\section{Study area}

Geographically, Nepal is divided into three regions: Western (west to $\left.83^{\circ} \mathrm{E}\right)$; Central $\left(83^{\circ} \mathrm{E}\right.$ to $\left.86^{\circ} 30^{\prime} \mathrm{E}\right)$; and Eastern Nepal (east to $86^{\circ} 30^{\prime} E$ ) (Banerji 1963; Stainton 1972). Our study area included seven districts of Western Nepal: Kalikot, Mugu, Jumla, Jajarkot, Rolpa, Rukum Easr and Rukum West, and Dolpa (Map 1) and three protected areas i.e. Rara National Park, Dhorpatan Hunting Reserve, and Shey-Phoksundo National Park in Western Nepal.

Map 1 Study Area

\section{Data collection}

The International Centre for Integrated Mountain Development's (ICIMOD) land use map (http://geoportal.icimod.org/) was used to identify forest cover area within elevation range: $2000 \mathrm{~m}$ to $4000 \mathrm{~m}$ in potential red panda habitat. The DEM $90 \mathrm{~m}$ resolution image was used for elevation. The identified study area was divided into 504 grids of $9.6 \mathrm{~km}^{2}$ based on animal maximum home range (Yonzon 1989) using the Geospatial Modeling Environment built-in ArcGIS 10.2 version.

We selected $50 \%$ of grids which were further divided into 6 sub-grids (Area=1.6 $\mathrm{km}^{2}$ ). Altogether, 252 subgrids were selected randomly for sampling across the habitat. We followed the red panda field survey and protocol for community base monitoring for data collection (MoFSC 2015). Ensuring these selected sub-grids cover the entire potential habitat, including elevation range and water availability, in the particular grid. All the available transects with an average length of $1 \mathrm{~km}$ at an interval of $100 \mathrm{~m}$ contour were traversed in each sub-grid (MoFSC 2015). We recorded the red panda presence evidences based on indirect signs, such as droppings, foot prints, foraging sign and remains of dead body parts, and direct sighting while walking along the transects. Additionally, we also recorded the occurrence data opportunistically when encountered beside designed transects. Additionally, the habitat variables were collected in a concentric sampling plots with a radius $10 \mathrm{~m}$. Such sampling plots were also established in the red panda sign/sighting recorded site. Tree canopy cover and bamboo cover within a subplot of $10 \mathrm{~m}$ radius $\left(\mathrm{A}=314.28 \mathrm{~m}^{2}\right)$ and $1 \mathrm{~m}$ radius $\left(3.14 \mathrm{~m}^{2}\right)$ respectively also were recorded (MoFSC 2015). In total, we covered 1213 plots including 970 occurrence and 243 non-occurrence plots along $100.68 \mathrm{~km}$ long transects. All the field survey was conducted in June-July and September-October in 2016.

\section{Data filtering and processing}

Mostly, fecal pellets of red panda scat were used as indicative evidence of Himalayan red panda presence. The occurrence records $(\mathrm{n}=331)$ were used to predict distribution of Himalayan red panda using species distribution modeling techniques. Also, we categorized other field-collected data into three separate groups: topographic, habitat, and disturbance variables. All data were imported into excel spreadsheets and further statistical analysis was performed in $\mathrm{R}$ (Lê et al. 2008). Species absence record was nine times higher than the presence record, which could influence further statistical analysis. To address this inconsistency, we excluded those records consisting of more than $80 \%$ zero input values, and elevation below $2000 \mathrm{~m}$ and above $4000 \mathrm{~m}$ in further analysis.

Red pandas are relatively more abundant within these altitudinal range of (Choudhury 2001; Pradhan et al. 2001; Yonzon et al. 1991). At the same time, we also removed all the outliers from the data. 


\section{Potential habitat and corridors}

Occurrence data of red panda and bamboo species were extracted from the vegetation survey and used for distribution modeling based on the Maximum Entropy Algorithm (MaxEnt 3.3.3k). All 19 bioclimatic variables (11 temperature and 8 precipitation metrics) were downloaded from the WorldClim website (http://www.worldclim.org) (Hijmans et al. 2005). Our data were spatially distributed covering entire Western Nepal. All variables were converted into the ascii raster images with a cell size of 30 arc seconds $(\sim 1 \mathrm{~km})$ and masked by study area boundary for the modeling process. We run 5000 repetitions with a convergence threshold of 0.00001 , a regularization multiplier of 1 , a maximum number of 100,000 background points, the output grid format as "logistic," algorithm parameters set to auto features, and all other parameters at their default settings. Random test percentage was $25 \%$ of presence locations to test the performance of the model. The model outcome was evaluated by the Area Under Curve (AUC) of the Receiver-Operating Characteristic (ROC) plot. The training and test AUC above 0.75 indicated a reasonable to high model discrimination ability and good model performance (Elith et al. 2006). The habitat suitability map was built by combining the habitat model, bamboo distribution model, and forest cover using raster calculator in ARC GIS 10.2. We reclassified the habitat into three suitability classes: low (0.10.50), moderate (0.50-0.75), and high (>0.75) (Shrestha \& Bawa 2014; Thapa et al. 2018). Forest and bamboo habitat within $2000 \mathrm{~m}$ and $4000 \mathrm{~m}$ that consist river/stream, (sign recoded with 0.5 ), occurrence of low human footprint (https://sedac.ciesin.columbia.edu/data/set /wildareas-v2-human-footprint-geographic), and away from human settlements (excluded cattle sheds) (http://sedac.ciesin.columbia.edu/data/set/gpw-v4population-count-rev11), and habitat patches $\left(>9.6 \mathrm{~km}^{2}\right)$ were considered as an area of potential corridor. All these layers were built and overlay using a spatial analysis tool in ArcGIS 10.2.

Figure 1 Modeling process for building potential habitat for the red panda

\section{Relative abundance and habitat association}

Red pandas are elusive thus, it is hard to estimate their population size through direct sightings. So, the Encounter Rate (ER) of their signs was used as a standard method to measure their relative abundance (Ghose \& Dutta 2010; Bista et al. 2017). We calculated the ER as number of red panda signs per km. Habitat associated variables including topographic and vegetation covariates were analyzed using Principal Component Analysis (PCA) in FactoMineR package (Le et al. 2008).

\section{Results}

\section{Sign abundance and potential distribution}

We recorded the presence evidence of red panda from 331 plots in the eight districts in the western Nepal (Table1and Fig.2). A total of 331 (98.51\%) indirect signs and 5 (1.49\%) direct signs were recorded. An average ER of 3.34 signs $/ \mathrm{km}$ was recorded in the study area. The ER was significantly different in different districts. We recorded the highest ER in Jajarkot $(6.23 \mathrm{signs} / \mathrm{km})$ and least in Rolpa district $(0.94 \mathrm{signs} / \mathrm{km}$, Table 1).

Table 1 Relative abundance of red panda

\begin{tabular}{lllll}
\hline Districts & No. of Sign & Transect surveyed (no.) & Length of transect $(\mathrm{km})$ & ER $($ signs $/ \mathrm{km})$ \\
\hline Jajarkot & 181 & 48 & 29.00 & 6.23 \\
Dolpa & 37 & 26 & 8.54 & 4.33 \\
Rukum-East & 14 & 17 & 3.06 & 4.75 \\
Rukum-West & 10 & 12 & 3.00 & 3.33 \\
Mugu & 43 & 36 & 20.05 & 2.14
\end{tabular}




\begin{tabular}{lllll}
\hline Districts & No. of Sign & Transect surveyed (no.) & Length of transect $(\mathrm{km})$ & ER $($ signs $/ \mathrm{km})$ \\
\hline Kalikot & 11 & 18 & 5.19 & 2.10 \\
Jumla & 32 & 27 & 23.36 & 1.36 \\
Rolpa & 8 & 12 & 8.48 & 0.94 \\
Total & $\mathbf{3 3 6}$ & $\mathbf{1 9 6}$ & $\mathbf{1 0 0 . 6 8}$ & $\mathbf{3 . 3 4}$ \\
\hline
\end{tabular}

The training AUC and test AUC of the distribution model of red panda were 0.96 and 0.94 respectively. Similarly, the training AUC and test AUC of the potential bamboo species. were 0.93 and 0.92 respectively (Fig. 2). Both training and test AUC were higher than the random that indicated the model performed better than random. In western Nepal, potential habitat of red panda and bamboo distribution area were estimated to be $3,222 \mathrm{~km}^{2}$ and $6,294 \mathrm{~km}^{2}$ respectively. At total of $\mathrm{km}^{2}$ area was estimated as the potential habitat when combing overlapping area among distribution of Himalayan red panda, bamboo species and forest cover. (Table 2). The distribution model predicted more than two third of the total habitat available in only three districts, namely Jajarkot, Jumla and Kalikot, while the remaining five districts constituted less than one third of the habitat (Table 2).

Table 2 Potential suitable habitat for red panda based on predicted red panda habitat, bamboo distribution, and forest cover

Figure 2 Potential suitable habitat for bamboo sp. (left red color indicates potential range of bamboo) and red panda (right: green color indicates potential ranges of red panda)

This predicted habitat was found to be fragmented into numerous patches. The four districts: Jajarkot, Jumla, Mugu and Dolpa seemed to have conspicuous fragmentation than other four districts located in the east, which indicates the increasing fragmentation trend towards the west. (Fig.3).

Figure 3 Suitable habitat patches and possible corridors

\section{Habitat association}

We recorded the presence evidences between the elevation range of $2600 \mathrm{~m}$ to $3600 \mathrm{~m}$ (Fig. 5). Out of those, $75 \%$ signs were recorded between $3250 \mathrm{~m}$ and $3400 \mathrm{~m}$. Likewise, we recorded most of these signs in North-West aspect (23.6\%) followed by West (19.1\%), North-East (15.8\%) and East (14.5\%) aspects (Fig. 4). Their least preference was observed in Southern aspects $(9.5 \%, \mathrm{p}=6.29 \mathrm{E}-17)$. We recorded $90 \%$ of these presence signs within $150 \mathrm{~m}$ of a water distance (overall mean \pm s.d. $=101.32+-43.16 \mathrm{~m}$ ), Fig. 4). Similarly, we observed a large proportion ( $\mathrm{xx} \%$ ) of sign within the slope of 31.14@ $\pm 5.18 @$ (Fig. 2). Altogether 278 fecal piles were observed on tree branches, fallen logs, ground, and rock. Trees were the most common substrate used for defecation $(62.21 \%)$ followed by the ground surface $(29.96 \%)$ and fallen logs $(8.70 \%)$ and least in the rocks. (Fig. 2).

Figure 4 Distribution of sign records in different districts (left); record of sign along elevation in all study sites (right)

Figure 5 Records of red panda sign based on slope (right); records of red panda sign-based distance to water

The highest eigenvalue obtained showed that seven dimensions were required to capture all the data variations. By visualizing the scree plot and variance, at least five dimensions were required to retain $80 \%$ of the variance in the data (Fig.7). The first two dimensions of this space were plotted to examine the association among the variables in the red panda habitat. Dimension 1 accounted for $21.5 \%$ of the variance in the data and dimension 2 accounted for $18.8 \%$ of the variance (Fig. 6). Canopy cover, aspect and bamboo cover explained over $60 \%$ with other variables having low contribution in the first dimension. Similarly, the fallen 
log, slope, and elevation had high contribution in the second dimension. The analysis showed that these are the key micro-habitat variables influencing the distribution of red panda.

Table 3 Principle Component scores for PCA of habitat variables

Figure 6 Contribution of variables to Dim.1 and Dim. 2 (left and right)

Figure 7Bi-plot of principal component analysis (PCA) showing the relationship of habitat variables(right); a scree plot consisting a graph of the eigenvalues/variances associated with components (left)

\section{Discussion}

The conservation status of the red panda is a barometer of regional conservation efforts for monitoring the integrity of the Eastern Himalayan Broadleaf and Conifer Eco-region (Williams 2003). This study represents a major advance on past sparse and the restricted study which has principally relied on historical anecdotal information. Using extensive field-based survey ever conducted in Western Nepal, spanning red pandas' range districts of Western Nepal, we demonstrate how bioclimatic variables can be used to identify potential suitable habitat along with primary habitat requirements, distribution and potential corridor for the red panda conservation.

Based on extensive field survey and predictive potential habitat suitability model using bioclimatic variables to build a Maxent habitat model of red panda in Western Nepal. Our major findings have been 1) Potentially suitable habitat in Western Nepal, 2) Important attributes that influence red panda presence and 3) Number of potential corridors in Western Nepal. Our result with regards to total potential red panda habitat, habitat preference and potential corridors in Western Nepal could be critical for setting appropriate management goals, monitoring effectiveness, informing policymakers, and other relevant stakeholders. Western Nepal harbors $3,222 \mathrm{~km}^{2}$ i.e. $23.38 \%$ according to Panthi et al. (2019) and $14.86 \%$ as per Thapa et al. (2020) of potential red panda habitat in Nepal. This might suggest that Western Nepal has suitable ecological variables such as bamboo cover, canopy cover, aspects and water body needed for red panda survival.

Our study concluded that tree and bamboo cover, proximity to the water body and aspect were the strongest predictor of red panda distribution which is supported by findings of previous studies (Thapa et al. 2020; Thapa et al. 2018; Bista et al. 2017; Dorji et al. 2011; Williams 2006; Pradhan et al. 2001; Yonzon \& Hunter 1991a). Tree canopy cover has structured an ideal microclimatic setting (e.g., temperature, relative humidity; Anhuf \& Rollenbeck 2001) which favored the better growth of the understory of bamboo (Thapa et al. 2018) and also provide shelter and protection (Thapa et al. 2020; Bista et al. 2019; Pradhan et al. 2001; Yonzon \& Hunter 1991a). Also, canopy cover contributes for the safety shelter away from the avian predators which could showed the high record of fecal pellets in the tree branches. Also, $62.21 \%$ of the red panda latrine site was recorded on tree branches. This might be due to as tree provides better shelter and safety from predators and easy movement from the branches of trees (Reid et al. 1991; Pradhan et al 2001; Thapa et al. 2020).

The presence of bamboos was observed in $85 \%$ of sign plots. This demonstrates the importance of bamboos as one of the fundamental parameters affecting red panda distribution (Yonzon \& Hunter 1991b; Fox et al. 1996; Williams 2004; Dorji et al. 2011). Bamboo species contributes more than $90 \%$ food of the Himalayan red panda (Thapa et al. 2020; Panthi et al. 2019; Sharma et al. 2014 Pradhan et al. 2001; Yonzon \& Hunter 1989). Besides bamboo species, other food such as seasonal fruiting berries contribute a very little proportion in food of the red pandas (Yonzon \& Hunter 1991b). Such a near inclusive reliance on bamboo may be a survival strategy. Proximity to bamboo lessens the travel needed to forage, which conserve red panda energy (Hu 2001; Reid et al. 1991).

Occurrence of Himalayan red panda is positively associated with distance to water resources. Almost 90 of red panda fecal pellets were recorded within $150 \mathrm{~m}$ which might suggested that red panda frequently need 
water for their physiological food processing due to poor digestive system. Our findings are identical to the results of previous studies (Yonzon \& Hunter 1991b; Pradhan et al. 2001; Williams 2006; Dorji et al. 2011; Bista et al. 2017; Thapa et al. 2018). Such proximity might help the red panda to avoid predators such as snow leopard, marten, and human and conserve its energy (Pradhan et al. 2001; Bista et al. 2017). Proximity to the water is important to conserve their energy as they do not require a long distance to travel for water (Bista et al. 2017; Pradhan et al. 2001). Also, this might be also due to low water content associated with bamboo leaves (Reid et al. 1991; Wei et al. 1999b). In Wolong Reserve, the water content in their scat was $72.4 \pm 5.5 \%$, and that in B. faberi leaves $59.7 \pm 8.1 \%$ or less than $12.7 \%$ less than in the scat. (Johnson et al.1988). In the wild, their traces, including fecal pellets, foraging sites were frequently found at a site close to the water body. For instance, in Mabian Reserve, red pandas often foraged at sites less than $200 \mathrm{~m}$ away from the water body (Wei et al. 1999a). In CHAL, observation indicated that proximity to water may be an important habitat requirement because $90 \%$ of the fecal pellets were found within 100 $\mathrm{m}$ of the nearest water body (Bista et al. 2017).

Red pandas were detected more frequently on northerly and westerly slopes in our study, which supports similar observations by Yonzon (et al. 1991a) in Langtang National Park. Yonzon (et al. 1991a) hypothesized that the Northern slope receives fewer sunlight periods which are conducive to the growth of fir-jhapra bamboo forest. In Jigme Dorji and Thrumshingla National Parks, Bhutan, red pandas were associated strongly associated with southerly slopes as southerly slope receives direct sunlight during winter and red pandas rested in direct sunlight during winter to reduce heat loss (Reid et al. 1991). We noted that northerly and westerly slopes in our study areas had relatively high bamboo densities, which might be associated with sunlight and rainfall. Due to the lack of detailed knowledge of bamboo ecology in Nepal, more studies are needed to investigate the effects of the physical landscape variables on bamboo species.

In Nepal, forests are integral to human subsistence, however, because of the common reliance on forests by humans and red pandas, sustainable management of natural resources is critical to meet the needs of both people and red panda conservation. This proposes that red pandas are primarily dependent on bamboo species. Bamboo species are vulnerable to climate change because of their uncommon reproduction intervals (Janzen 1976), apart from limited seed dispersal capacity (Taylor et al. 1991; Mao Ning et al. 2012). To ensure red panda survival bamboo conservation should be the highest importance. Therefore, the establishment of red panda focused conservation zones is needed to secure the long-term survival of red pandas through maintaining habitat connectivity that ensures the conservation of a genetically viable population in the long run. This study recommended potential five potential corridors in western Nepal. Although, a detailed field-based assessment is needed to validate it scientifically.

The present study identified five potential corridors in Western Nepal with an important cluster in Jumla, Jajarkot, and Dolpa district. These corridors are the vital repository of biodiversity in this region. Although, biodiversity of the suggested corridor has not been affected by any detrimental development activities, the same cannot be forecast for the future. It is imperative that the partnerships and collaborations with the community should be nurtured to ensure the long-term conservation of suggested the corridor. This study also further highlighted the need for site-specific conservation strategies that need to be bolstered with comprehensive information on bamboo distribution, other palatable species, and microclimatic conditions.

\section{Data Accessibility Statement}

We will share our data via Dryad public repository after the acceptance of the manuscript for publication. 


\section{Reference}

Anhuf, Dieter, and RütgerRollenbeck. "Canopy structure of the Rio Surumoni rain forest (Venezuela) and its influence on microclimate." Ecotropica 7 (2001): 21-32.

Banerji, Mohan Lal. "Outline of Nepal phytogeography." Vegetatio 11, no. 5-6 (1963): 288-296.

Bahuguna, C., S. Dhaundyal, P. Vyas, and N. Singhal. "The red panda at Singalila National Park and adjoining forest: a status report." Small Carnivore Conservation 19, no. 1 (1998): l-12.

Bhatta, Manoj, Karan Bahadur Shah, Bhupendra Devkota, Rajiv Paudel, and Saroj Panthi. "Distribution and habitat preference of red panda (Ailurus fulgens fulgens) in Jumla district, Nepal." Open Journal of Ecology 4, no. 15 (2014): 989.

Bista, Damber, Saroj Shrestha, Peema Sherpa, Gokarna Jung Thapa, Manish Kokh, Sonam Tashi Lama, Kapil Khanal, Arjun Thapa, and Shant Raj Jnawali. "Distribution and habitat use of red panda in the Chitwan-Annapurna Landscape of Nepal." PloS one 12, no. 10 (2017).

Braun, Clait E. Techniques for wildlife investigations and management. Wildlife Society, 2005.

Choudhury, Anwaruddin. "An overview of the status and conservation of the red panda Ailurus fulgens in India, with reference to its global status." Oryx 35, no. 3 (2001): 250-259.

DNPWC, DFSC. "Red Panda Conservation Action Plan for Nepal (2019-2023)". Department of National Parks and Wildlife Conservation and Department of Forests and Soil Conservation, Kathmandu, Nepal. (2018).

Dongol, B. Habitat and Distribution of Red Panda: A case from Ranchuli VDC Kalikot District, Nepal, A report submitted to Red panda Network, 2014, Kathmandu, Nepal. http://www.iucnredlist.org/details/ biblio/714/0. Downloaded on 29 December 2016.

Dorji, Sangay, Karl Vernes, and Rajanathan Rajaratnam. "Habitat correlates of the red panda in the temperate forests of Bhutan." PLoS One 6, no. 10 (2011): e26483.

Dorji S, Rajaratnam R, Vernes K. The red panda Ailurus fulgens in the Himalayan Kingdom of Bhutan: Distribution, conservation status, and management recommendations. Oryx in press

Dudik, Miroslav, S. J. Philips, and R. E. Shapire. "A maximum entropy approach to species distribution modelling." In Proceedings of the 21st International Conference on Machine Learning. 2004.

Elith*, Jane, Catherine H. Graham*, Robert P. Anderson, Miroslav Dudík, Simon Ferrier, Antoine Guisan, Robert J. Hijmans et al. "Novel methods improve prediction of species' distributions from occurrence data." Ecography 29, no. 2 (2006): 129-151.

Elith, Jane, Steven J. Phillips, Trevor Hastie, Miroslav Dudík, Yung En Chee, and Colin J. Yates. "A statistical explanation of MaxEnt for ecologists." Diversity and distributions 17, no. 1 (2011): 43-57.

Fox, Jefferson, Pralad Yonzon, and Nancy Podger. "Mapping conflicts between biodiversity and human needs in Langtang National Park, Nepal." Conservation Biology 10, no. 2 (1996): 562-569.

Franklin, Janet, Frank W. Davis, Makihiko Ikegami, Alexandra D. Syphard, Lorraine E. Flint, Alan L. Flint, and Lee Hannah. "Modeling plant species distributions under future climates: how fine scale do climate projections need to be?" Global change biology 19, no. 2 (2013): 473-483.

Ghose, Dipankar, and Pijush Kumar Dutta. "Status and distribution of Red Panda Ailurus fulgensfulgens in India." In Red Panda, pp. 357-373. William Andrew Publishing, 2011. 
Glatston, Angela R. "Red panda biology." In Red Panda Conference (1987: Rotterdam Zoo). SPB Academic Publishing, 1989.

Glatston, Angela R. The red panda, olingos, coatis, raccoons, and their relatives: status survey and conservation action plan for procyonids and ailurids. IUCN, 1994.

Glatston, Angela R., ed. Red Panda: Biology and Conservation of the First Panda. William Andrew, 2010.

Glatston, Angela R., and Axel Gebauer. "People and Red Pandas: The Red Panda's Role in Economy and Culture." In Red Panda, pp. 11-25. William Andrew Publishing. 2011.

Glatston, A., F. Wei, Zaw THAN, and A. Sherpa. "Ailurus fulgens. The IUCN Red List of Threatened Species: e. T714A45195924." (2015).

Guisan, Antoine, and Niklaus E. Zimmermann. "Predictive habitat distribution models in ecology." Ecological modelling 135, no. 2-3 (2000): 147-186.

Guisan, Antoine, Wilfried Thuiller, and Niklaus E. Zimmermann. Habitat suitability and distribution models: with applications in R. Cambridge University Press, 2017.

Han ZX, Hu JC. Status and protection of the red panda. Bulletin of Biology. 2004; 39:7-9.

Hijmans, Robert J., Susan E. Cameron, Juan L. Parra, Peter G. Jones, and Andy Jarvis. "Very high-resolution interpolated climate surfaces for global land areas." International Journal of Climatology: A Journal of the Royal Meteorological Society 25, no. 15 (2005): 1965-1978.

Hu, J. C. (2001). Research on the giant panda. Shanghai, China: Shanghai Scientific and Technological Press. Hu, Yibo, Arjun Thapa, and Fuwen Wei. "Ailurus fulgens (Himalayan Red Panda) and Ailurus styani (Chinese Red Panda)." Trends in genetics: TIG (2020).

Janzen, Daniel H. "Why bamboos wait so long to flower." Annual Review of Ecology and systematics 7, no. 1 (1976): 347-391.

Jnawali, S., K. Leus, S. Molur, A. Glatston, and S. Walker. "Red panda (Ailurus fulgens)." In Population and Habitat Viability Assessment (PHVA) and Species Conservation Strategy (SCS) Workshop Report. National Trust for Nature Conservation, Kathmandu, Nepal, Conservation Breeding Specialist Group and Zoo Outreach Organization, Coimbatore, India. (2012).

Johnson, Kenneth G., George B. Schaller, and Hu Jinchu. "Comparative behavior of red and giant pandas in the Wolong Reserve, China." Journal of mammalogy 69, no. 3 (1988): 552-564.

Krebs, Charles J. Ecology: the experimental analysis of distribution and abundance/by Charles J. Krebs. No. 574.5 K74. 2009.

Lê, Sébastien, Julie Josse, and François Husson. "FactoMineR: An R package for multivariate analysis." Journal of statistical software 25, no. 1 (2008): 1-18.

Liu, Canran, Matt White, and Graeme Newell. "Selecting thresholds for the prediction of species occurrence with presence-only data." Journal of biogeography 40, no. 4 (2013): 778-789.

Mahato, N. K. "Status of red panda (Ailurus fulgens) in Kangchenjunga Region of Nepal." Tigerpaper 31 (2004): 7-9.

Ministry of Forests and Soil Conservation. "Red Panda Field Survey and Protocol for Community Based Monitoring". Ministry of Forests and Soil Conservation, Singha Durbar, Kathmandu, Nepal. (2015).

Mosaddad, Seyed Ali, Elahe Tahmasebi, Alireza Yazdanian, Mohammad Bagher Rezvani, Alexander Seifalian, Mohsen Yazdanian, and Hamid Tebyanian. "Oral microbial biofilms: an update." European Journal of Clinical Microbiology \& Infectious Diseases (2019): 1-15. 
Panthi, Saroj, Achyut Aryal, David Raubenheimer, Jennie Lord, and Bikash Adhikari. "Summer Diet and Distribution of the Red Panda (Ailurus fulgensfulgens) in Dhorpatan Hunting Reserve, Nepal." Zoological Studies 51, no. 5 (2012): 701-709.

Phillips, Steven J., Robert P. Anderson, and Robert E. Schapire. "Maximum entropy modeling of species geographic distributions." Ecological modelling 190, no. 3-4 (2006): 231-259.

Pradhan, Sunita, Gautom K. Saha, and Jamal A. Khan. "Ecology of the red panda Ailurus fulgens in the Singhalila National Park, Darjeeling, India." Biological Conservation 98, no. 1 (2001): 11-18.

Reid, D. G., Hu Jinchu, and Huang Yan. "Ecology of the red panda Ailurus fulgens in the Wolong Reserve, China." Journal of Zoology 225, no. 3 (1991): 347-364.

Sharma, Hari P., Jerrold L. Belant, and Pei-Jen L. Shaner. "Attitudes towards conservation of the Endangered red panda Ailurus fulgens in Nepal: a case study in protected and non-protected areas." Oryx (2017): $1-6$.

Sharma, Hari Prasad, Jon Swenson, and Jerrold L. Belant. "Seasonal food habits of the red panda (Ailurus fulgens) in Rara National Park, Nepal." (2014).

Southern Oscillation Index." Meteorology and Atmospheric Physics 75, no. 1-2 (2000): 21-28.

Sinclair, A. R. E., Fryxell, J. M., \&Caughley, G. (2006). Wildlife ecology, conservation, and management: Blackwell Pub.

Sinclair, Steve J., Matthew D. White, and Graeme R. Newell. "How useful are species distribution models for managing biodiversity under future climates?" Ecology and Society 15, no. 1 (2010).

Stainton, J. D. A. Forests of Nepal. London, UK: John Murray. (1972).

Taylor, Alan H., Donald G. Reid, Qin Zisheng, and Hu Jinchu. "Spatial patterns and environmental associates of bamboo (Bashaniafangiana Yi) after mass-flowering in Southwestern China." Bulletin of the Torrey Botanical Club (1991): 247-254.

Thapa, Arjun, Ruidong Wu, Yibo Hu, YonggangNie, Paras B. Singh, Janak R. Khatiwada, Li Yan, Xiaodong $\mathrm{Gu}$, and Fuwen Wei. "Predicting the potential distribution of the endangered red panda across its entire range using MaxEnt modeling." Ecology and evolution 8, no. 21 (2018): 10542-10554.

Tremlova, Kateřina, and Zuzana Münzbergová. "Importance of species traits for species distribution in fragmented landscapes." Ecology 88, no. 4 (2007): 965-977.

Tuanmu, Mao-Ning, Andrés Viña, Julie A. Winkler, Yu Li, Weihua Xu, Zhiyun Ouyang, and Jianguo Liu. "Climate-change impacts on understorey bamboo species and giant pandas in China's Qinling Mountains." Nature Climate Change 3, no. 3 (2013): 249-253.

Wei, Fuwen, Zuojian Feng, Zuwang Wang, and Jinchu Hu. "Current distribution, status and conservation of wild red pandas Ailurus fulgens in China." Biological conservation 89, no. 3 (1999a): 285-291.

Wei, F., Z. Feng, Z. Wang, and M. Li. "Feeding strategy and resource partitioning between giant and red pandas." Mammalia 63, no. 4 (1999b): 417-430.

Wei, Fuwen, Zuojian Feng, Zuwang Wang, and Jinchu Hu. "Habitat use and separation between the giant panda and the red panda." Journal of Mammalogy 81, no. 2 (2000): 448-455.

Wei, Fuwen, and Zejun Zhang. "Red panda ecology." In Red Panda, pp. 193-212. William Andrew Publishing, 2011.

Williams, Brian H. "Red panda in eastern Nepal: how do they fit into ecoregional conservation of the eastern Himalaya." Conservation Biology in Asia 16 (2003): 236-250. 
Williams, Brian H. "The status of the red panda in Jamuna and Mabu villages of eastern Nepal." PhD diss., San José State University. (2004).

Williams, Brian. "Red Panda Project." Earth Island Journal 21, no. 3 (2006): 18-18.

Wilson, Kerrie A., Josie Carwardine, and Hugh P. Possingham. "Setting conservation priorities." Annals of the New York Academy of Sciences 1162, no. 1 (2009): 237-264.

Wisz, Mary S., Mikkel P. Tamstorf, Jesper Madsen, and Martin Jespersen. "Where might the Western Svalbard tundra be vulnerable to pink-footed goose (Anser brachyrhynchus) population expansion? Clues from species distribution models." Diversity and Distributions 14, no. 1 (2008): 26-37.

Yonzon, P. B., and M. L. Hunter. "Ecological study of the red panda in the Nepal-Himalaya" Red panda biology 1, no. 7 (1989).

Yonzon, Pralad B., and Malcolm L. Hunter Jr. "Conservation of the red panda Ailurus fulgens ." Biological conservation 57, no. 1 (1991a): 1-11.

Yonzon, Pralad B., and Malcolm L. Hunter Jr. "Cheese, tourists, and red pandas in the Nepal Himalayas." Conservation Biology 5, no. 2 (1991b): 196-202.

\section{Tables}

Table 1 Relative abundance of red panda

\begin{tabular}{lllll}
\hline Districts & No. of Sign & Transect surveyed (no.) & Length of transect $(\mathrm{km})$ & ER $($ signs $/ \mathrm{km})$ \\
\hline Jajarkot & 181 & 48 & 29.00 & 6.23 \\
Dolpa & 37 & 26 & 8.54 & 4.33 \\
Rukum-East & 14 & 17 & 3.06 & 4.75 \\
Rukum-West & 10 & 12 & 3.00 & 3.33 \\
Mugu & 43 & 36 & 20.05 & 2.14 \\
Kalikot & 11 & 18 & 5.19 & 2.10 \\
Jumla & 32 & 27 & 23.36 & 1.36 \\
Rolpa & 8 & 12 & 8.48 & 0.94 \\
Total & $\mathbf{3 3 6}$ & $\mathbf{1 9 6}$ & $\mathbf{1 0 0 . 6 8}$ & $\mathbf{3 . 3 4}$ \\
\hline
\end{tabular}

Table 2 Potential suitable habitat for red panda based on predicted red panda habitat, bamboo distribution, and forest cover

\begin{tabular}{lll}
\hline District & Area $\left(\mathrm{km}^{2}\right)$ & Habitat $(\%)$ \\
\hline Jumla & 1,087 & 33.74 \\
Kalikot & 711 & 22.07 \\
Jajarkot & 516 & 16.01 \\
Rukum-East & 220 & 6.82 \\
Rukum-West & 120 & 3.72 \\
Dolpa & 251 & 7.79 \\
Mugu & 248 & 7.70 \\
Rolpa & 69 & 2.14 \\
Total & $\mathbf{3 , 2 2 2}$ & $\mathbf{1 0 0 . 0 0}$ \\
\hline
\end{tabular}


Table 3 Principle Component scores for PCA of habitat variables

\begin{tabular}{llll}
\hline Variables & Dim.1 & Dim.2 & Dim.3 \\
\hline Elevation & -0.133 & -0.466 & 0.606 \\
Aspect & 0.687 & -0.02 & 0.25 \\
Slope & 0.261 & -0.55 & -0.516 \\
Canopy_cov & 0.691 & 0.017 & -0.352 \\
Bamb_cov & 0.632 & 0.34 & 0.303 \\
Dist_water & -0.263 & 0.429 & -0.372 \\
Fallen_Log & -0.026 & 0.706 & 0.094 \\
\hline
\end{tabular}

\section{Maps and Figures}

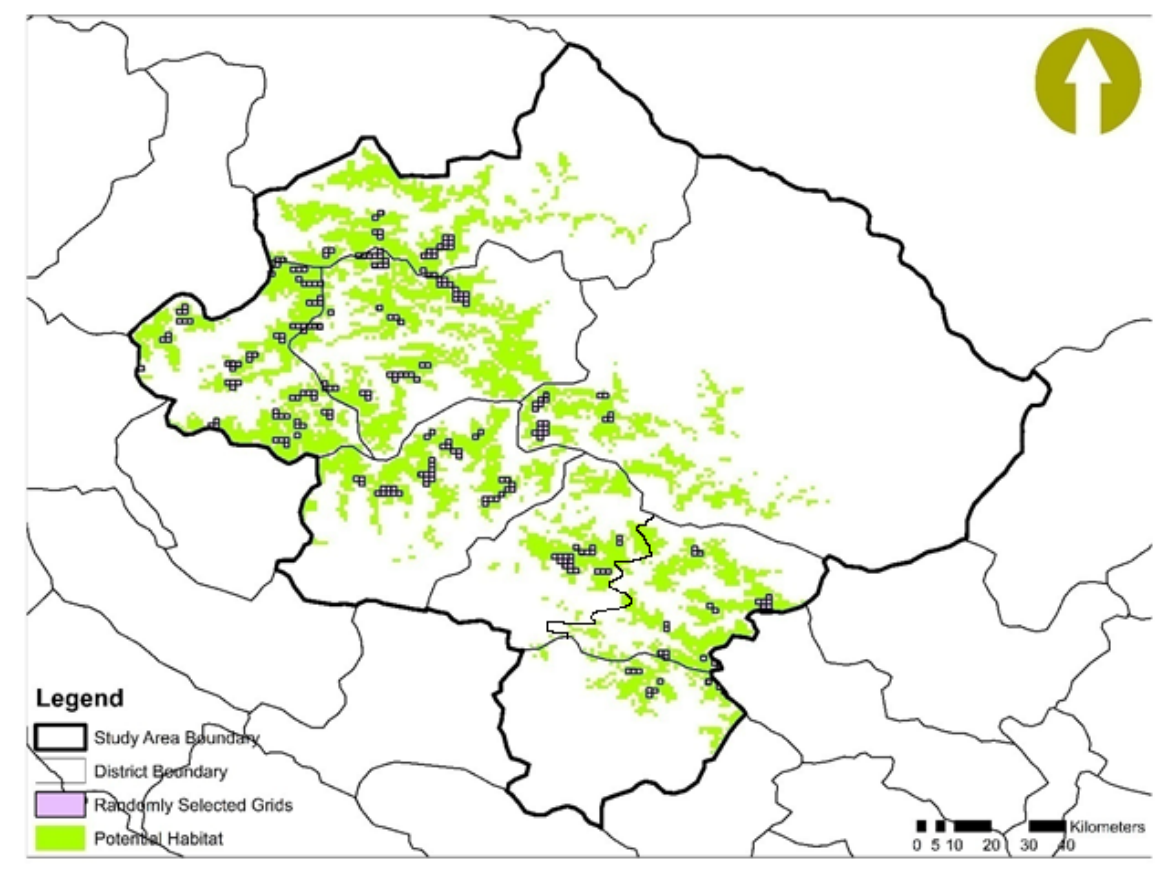

Map 1 Study Area 

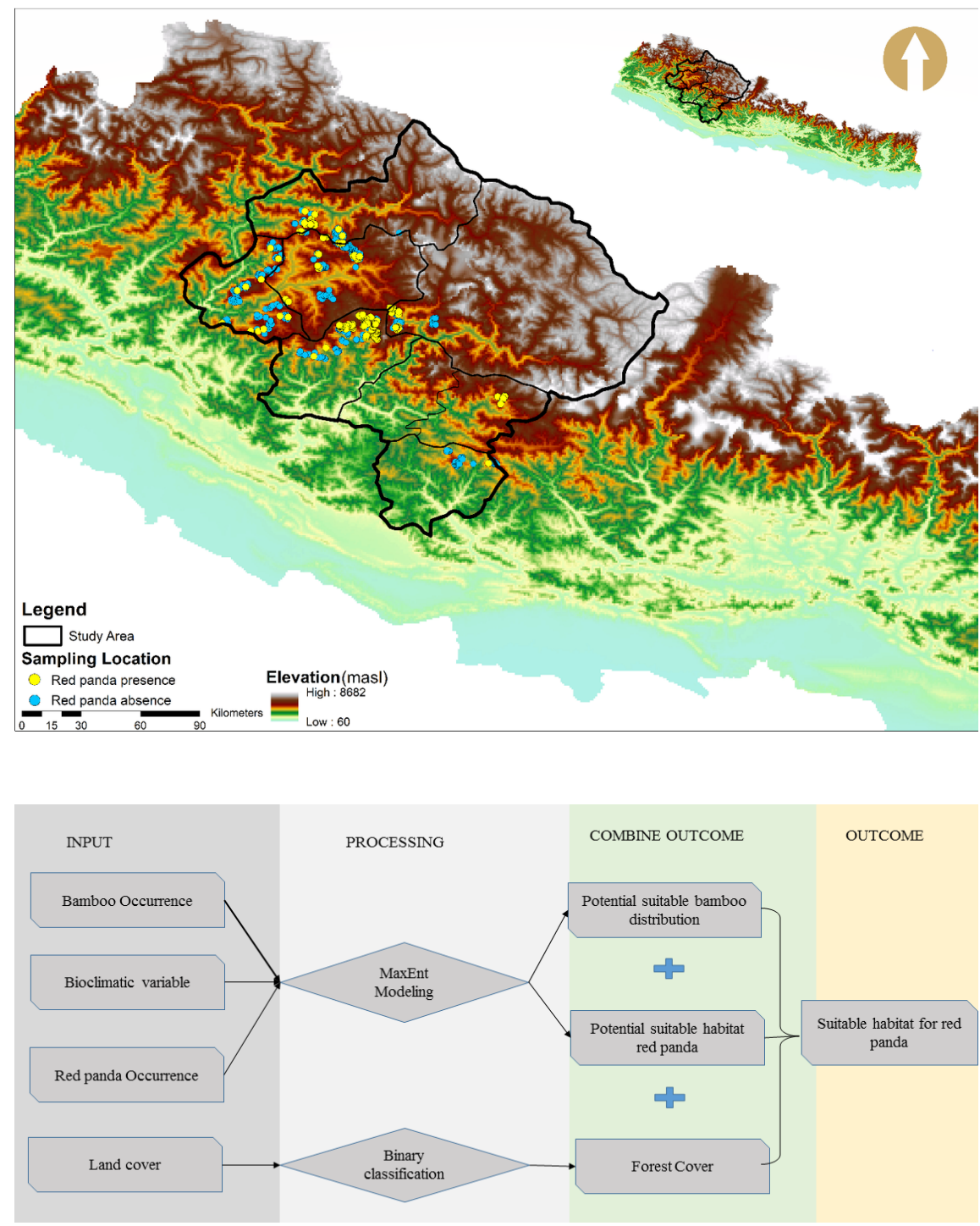

Figure 1 Modeling process for building potential habitat for the red panda 


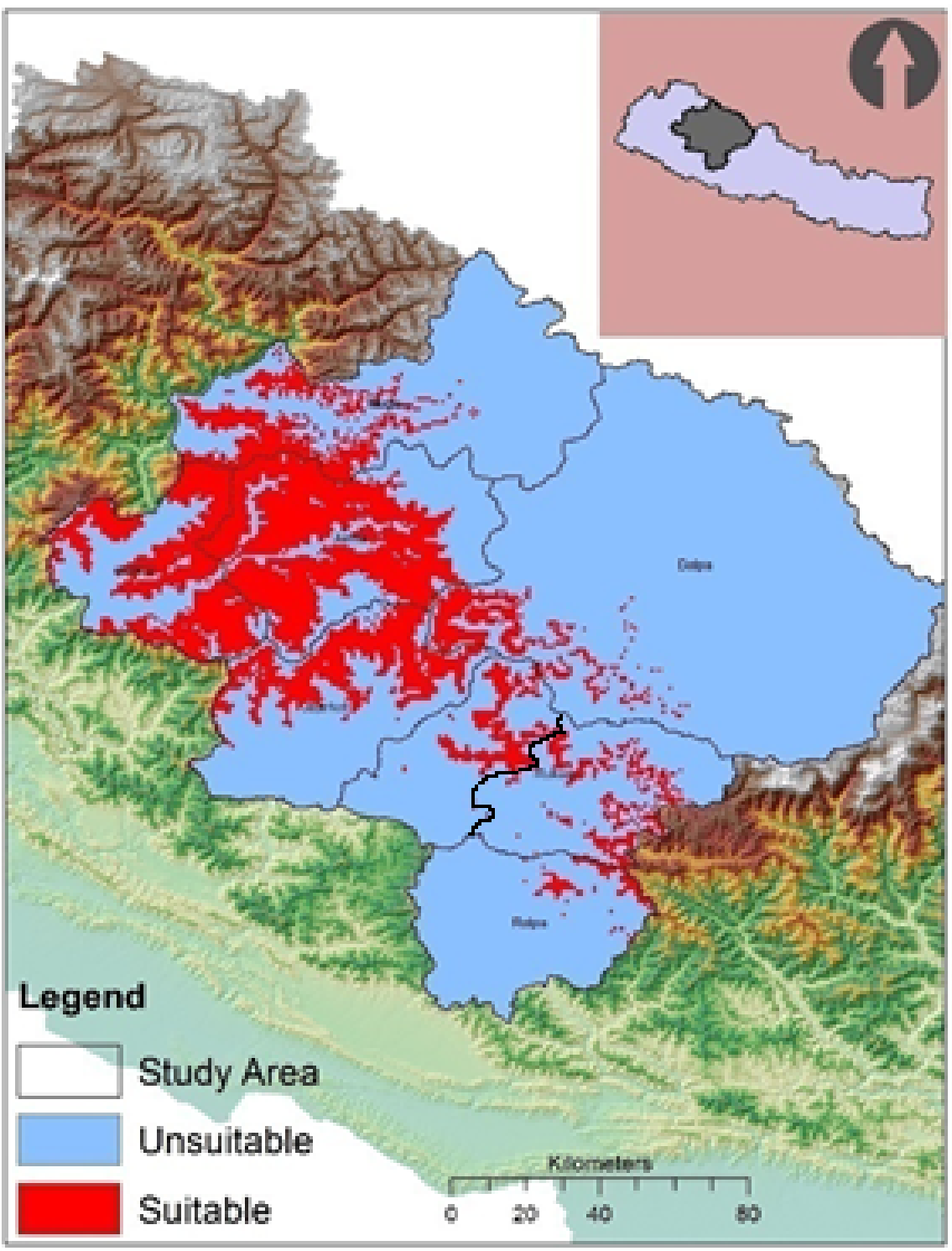




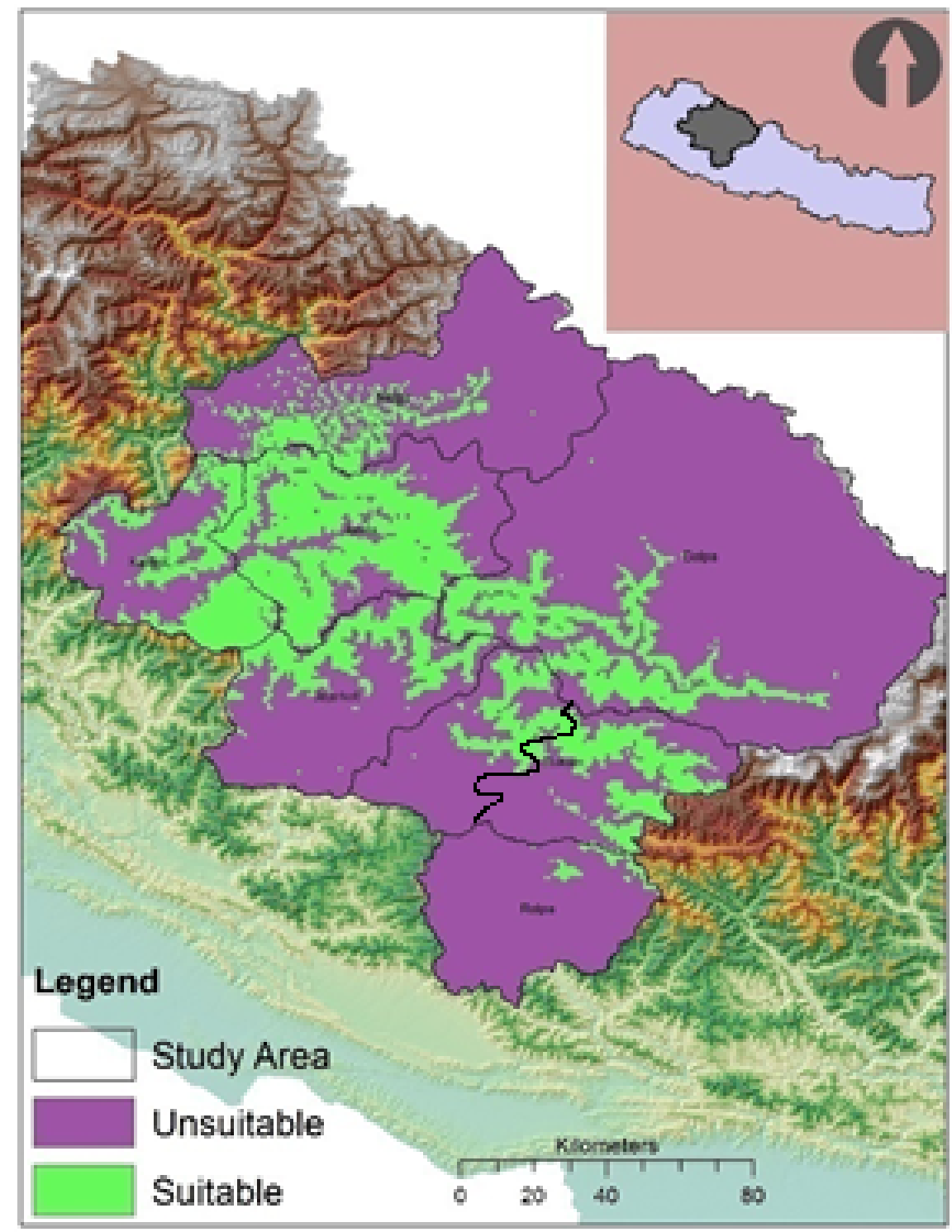

Figure 2 Potential suitable habitat for bamboo sp. (left red color indicates potential range of bamboo) and red panda (right: green color indicates potential ranges of red panda) 


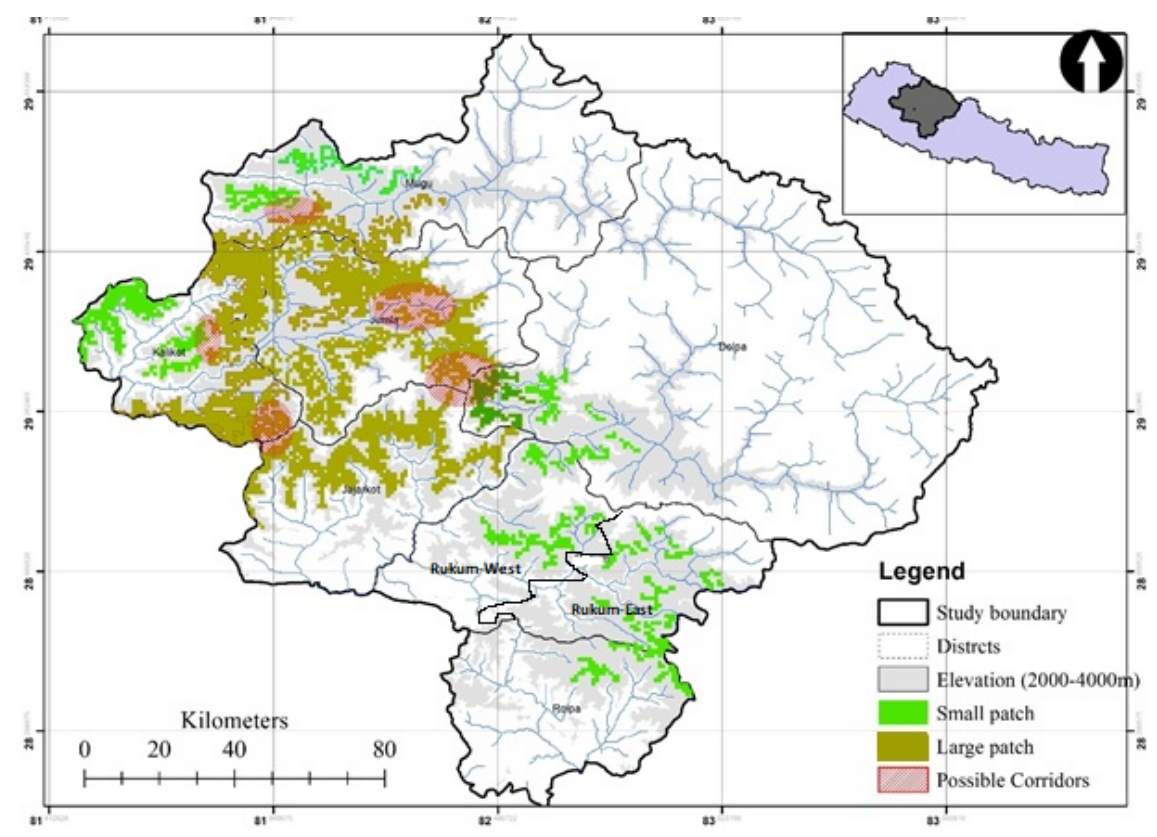

Figure 3 Suitable habitat patches and possible corridors

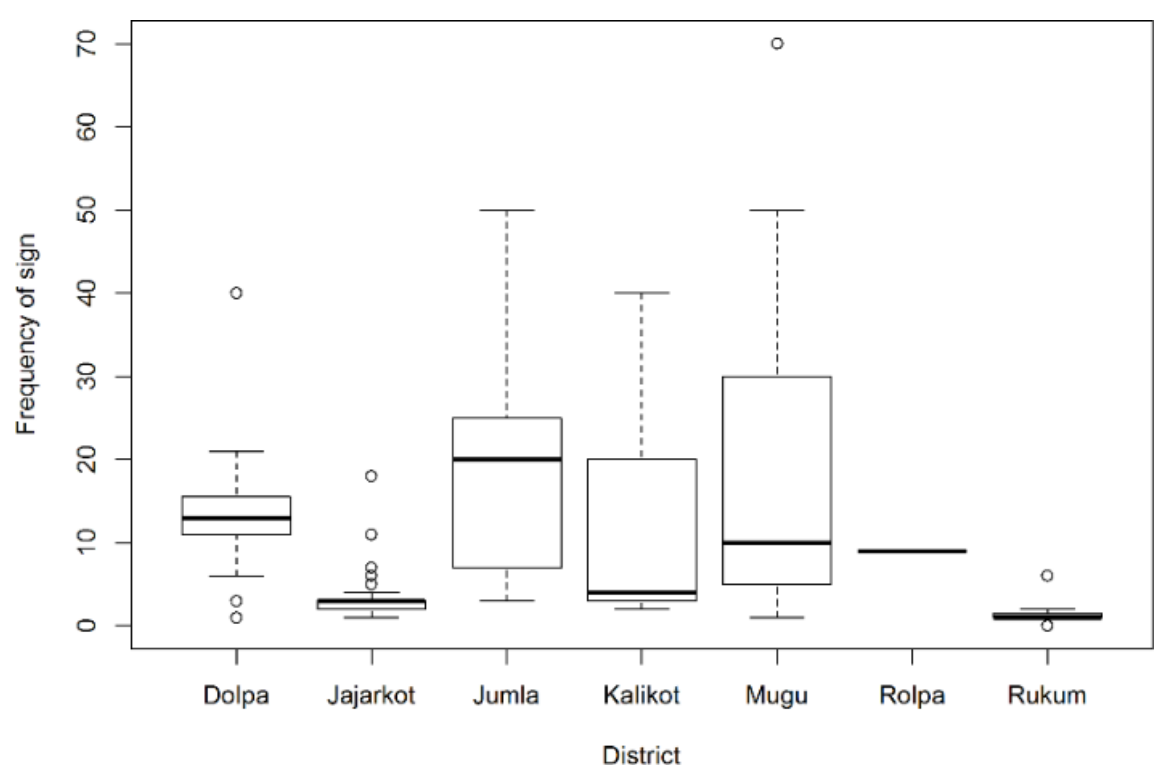




\section{Elevation and sign frequency}

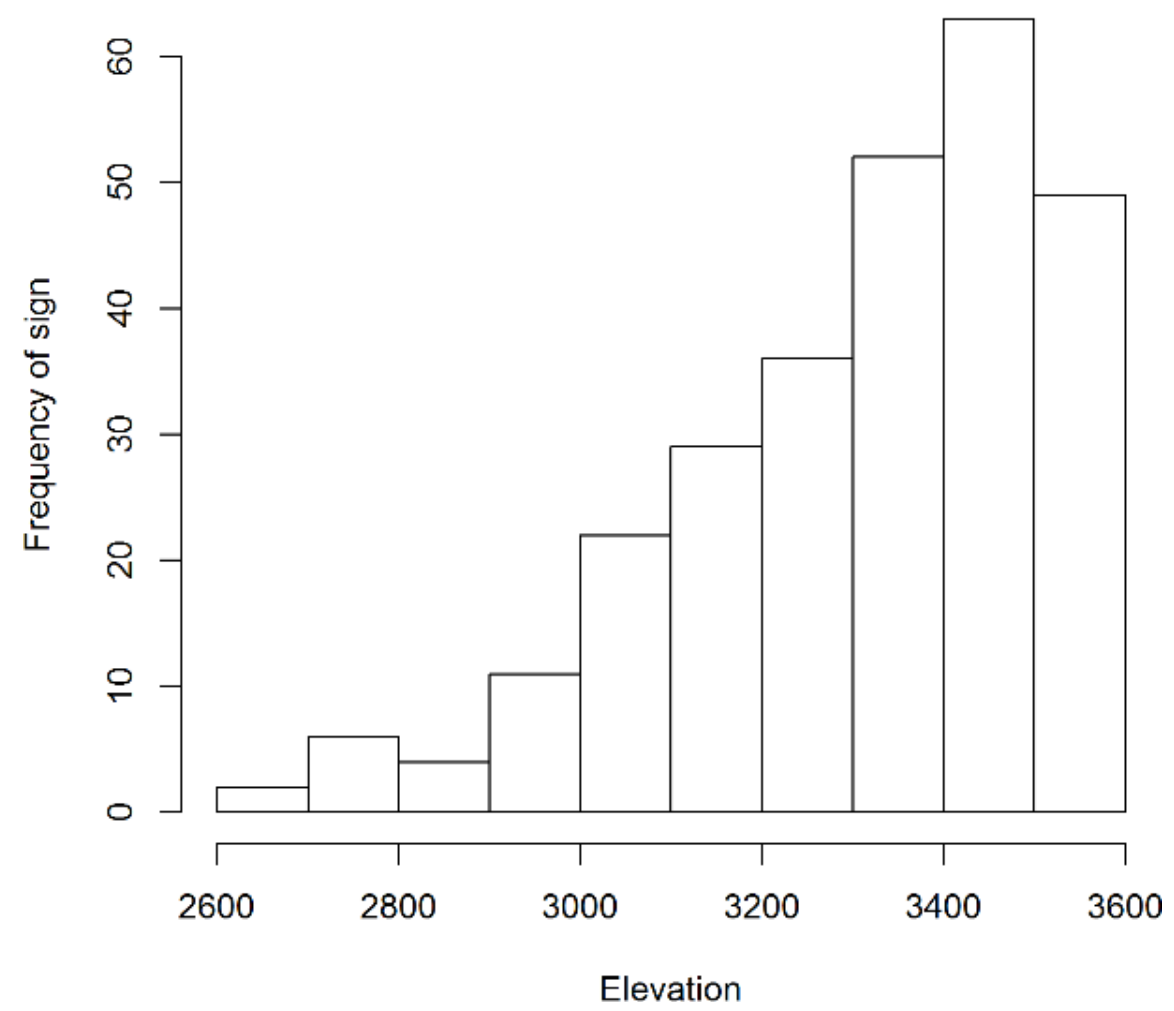

Figure 4 Distribution of sign records in different districts (left); record of sign along elevation in all study sites (right) 
Slope and sign frequency

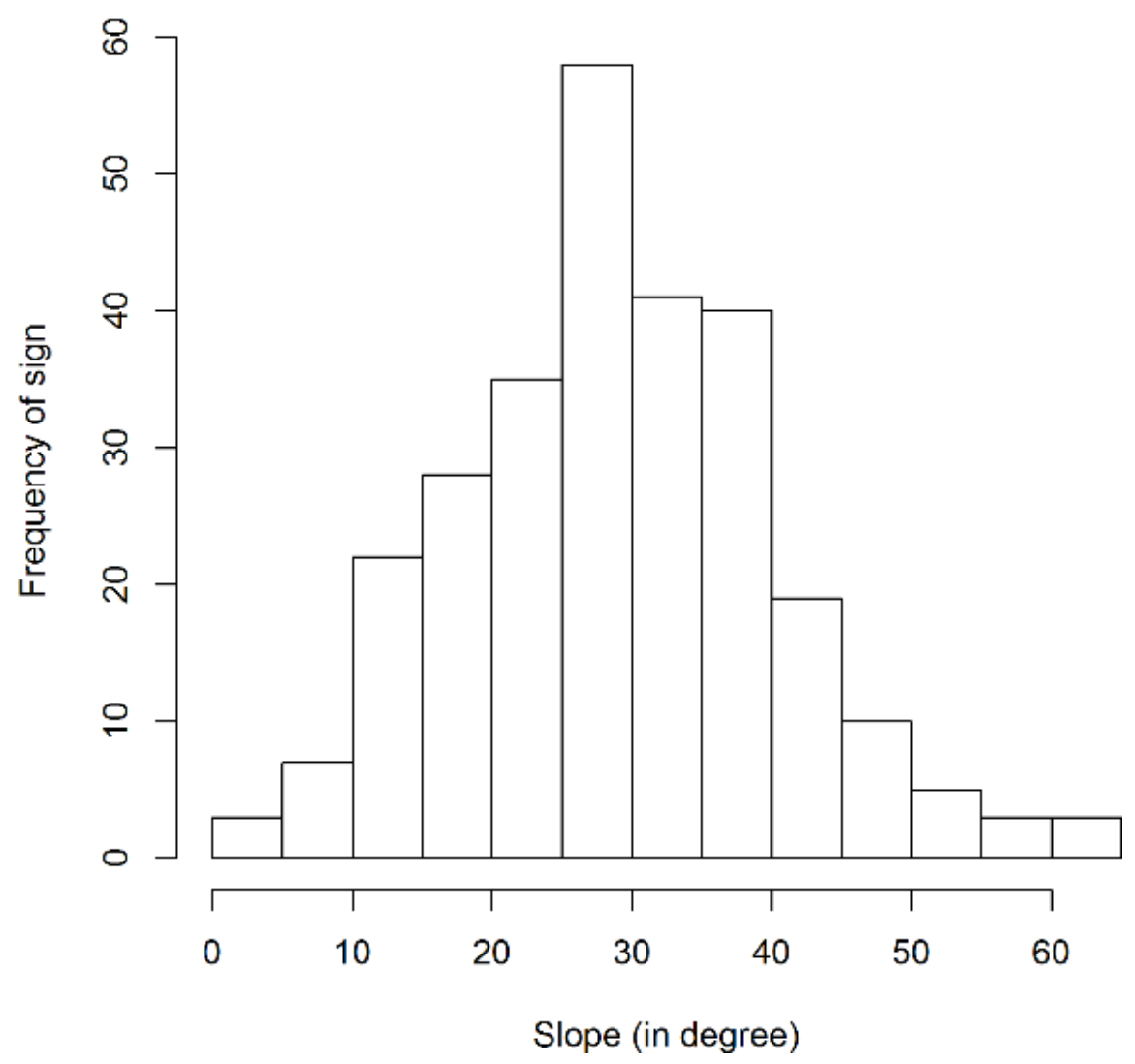




\section{Distance to water and sign frequency}

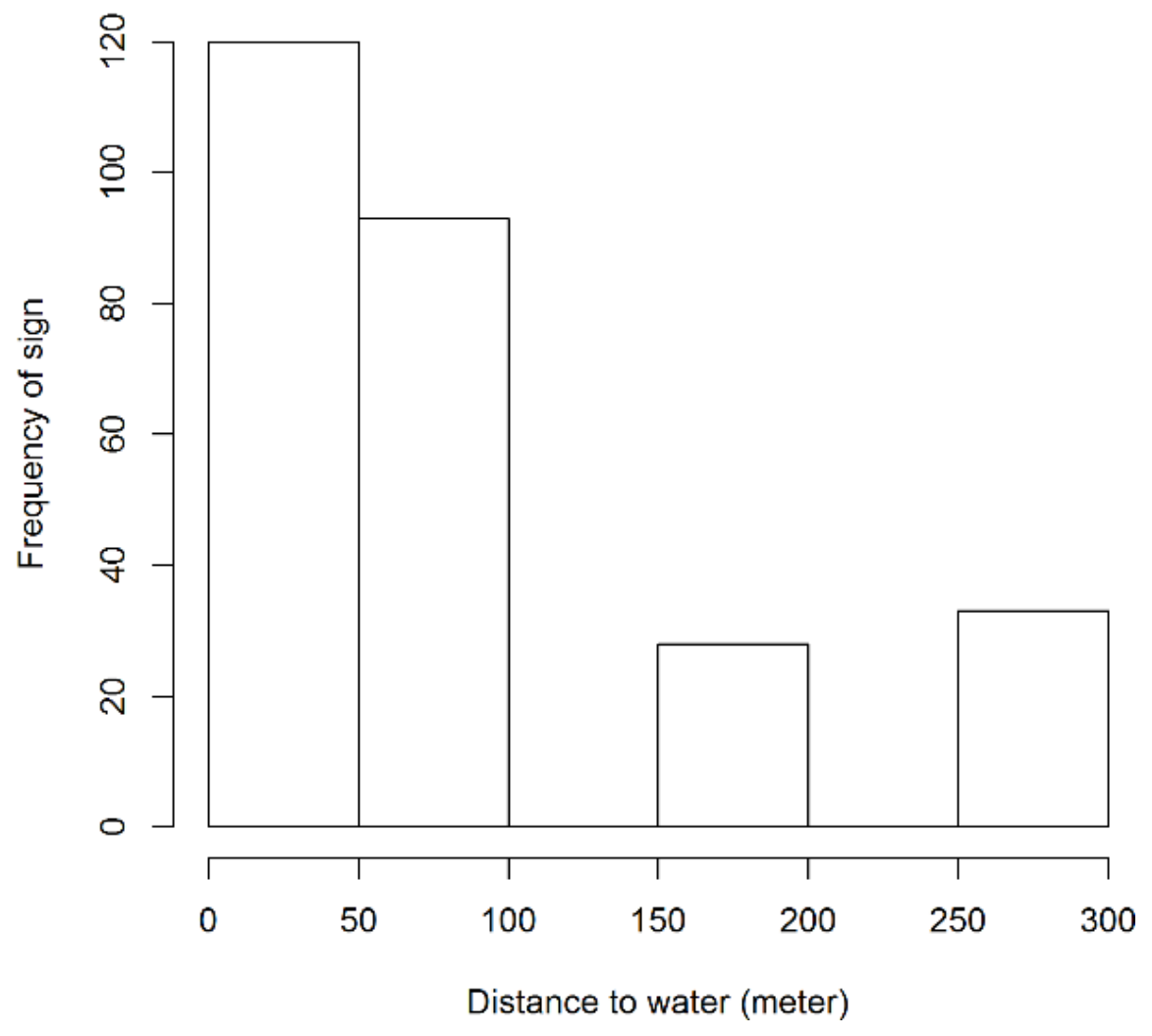

Figure 5 Records of red panda sign based on slope (right); records of red panda sign-based distance to water 


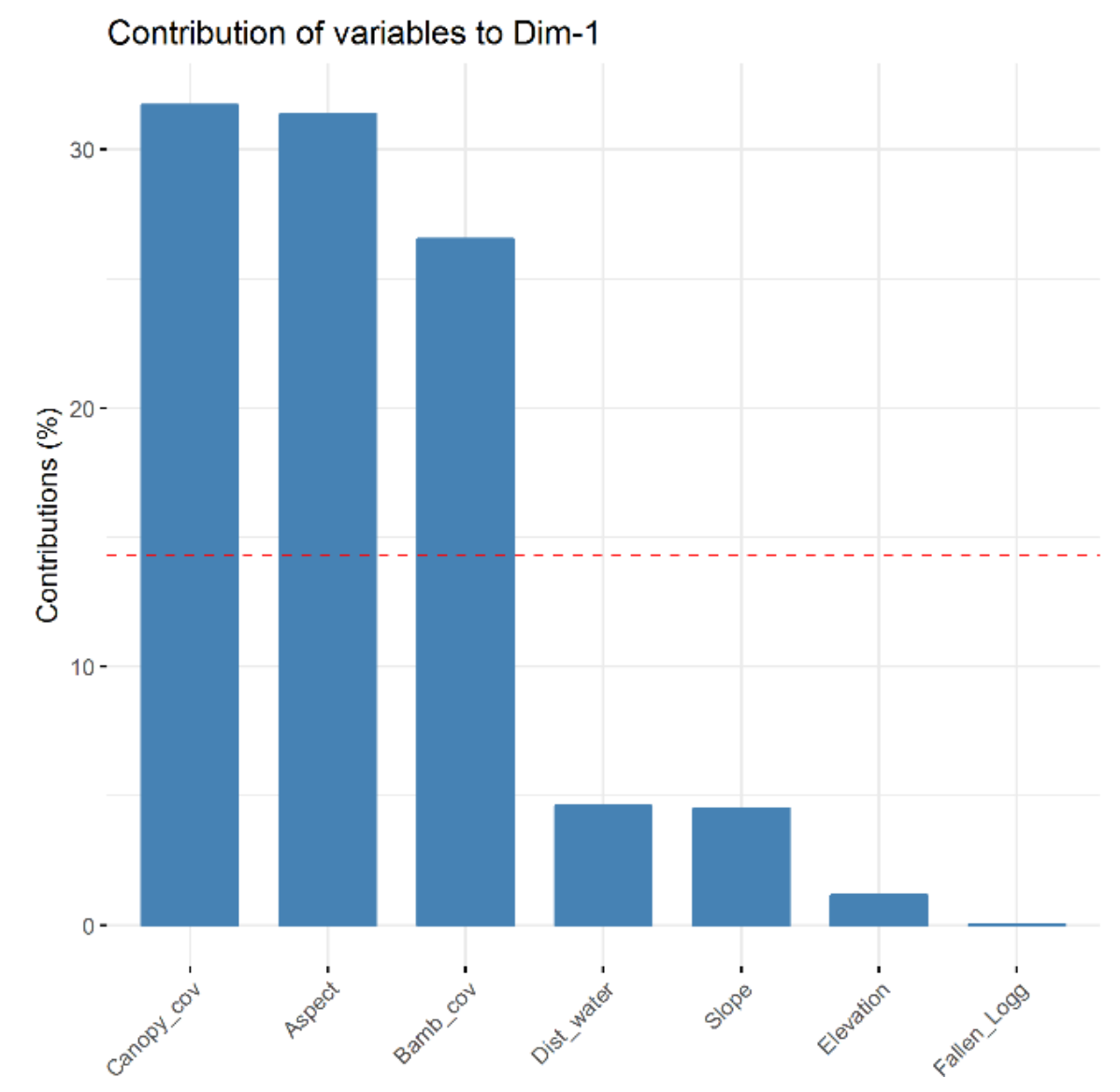


Contribution of variables to Dim-2

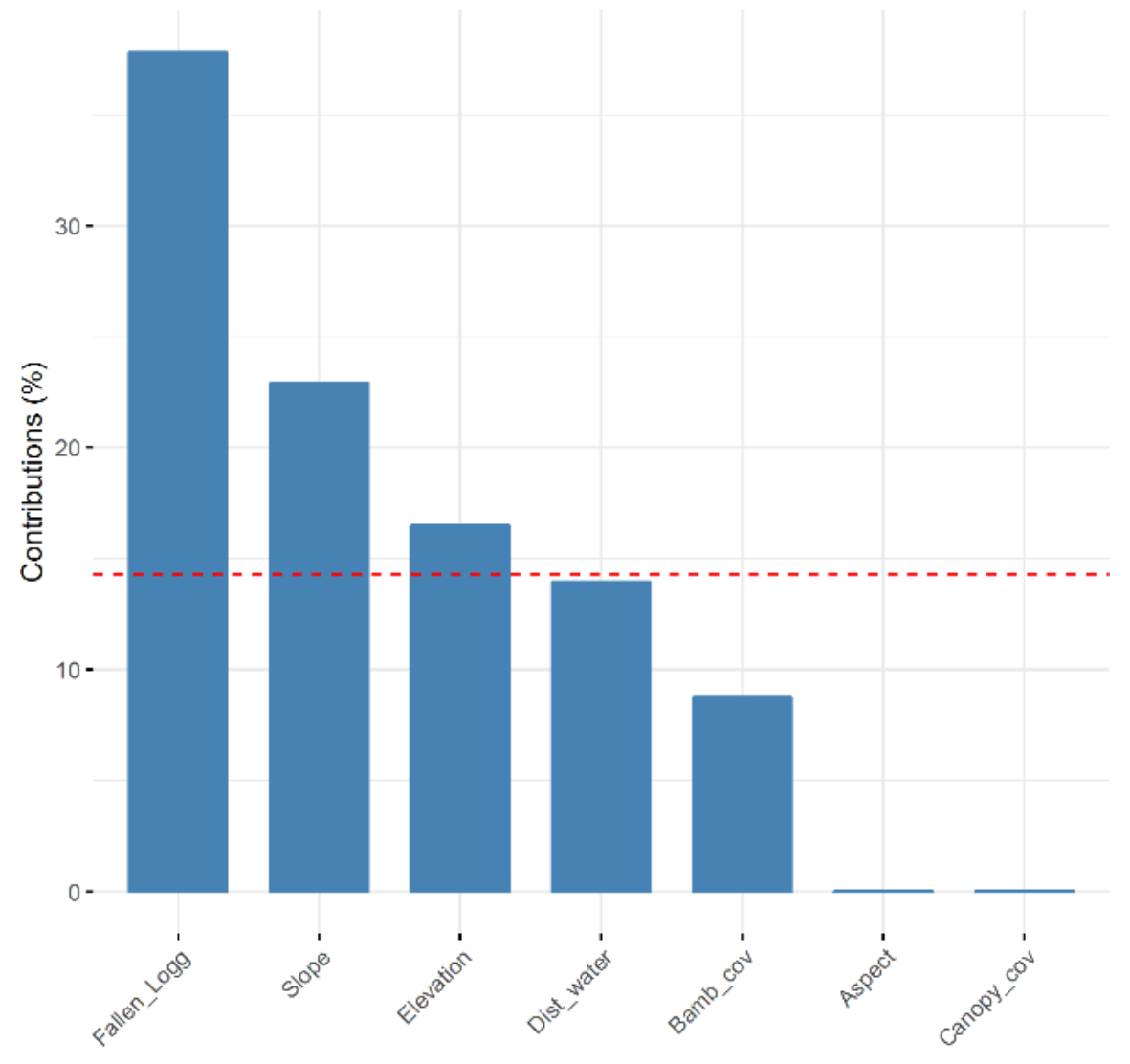

Figure 6 Contribution of variables to Dim.1 and Dim. 2 (left and right) 


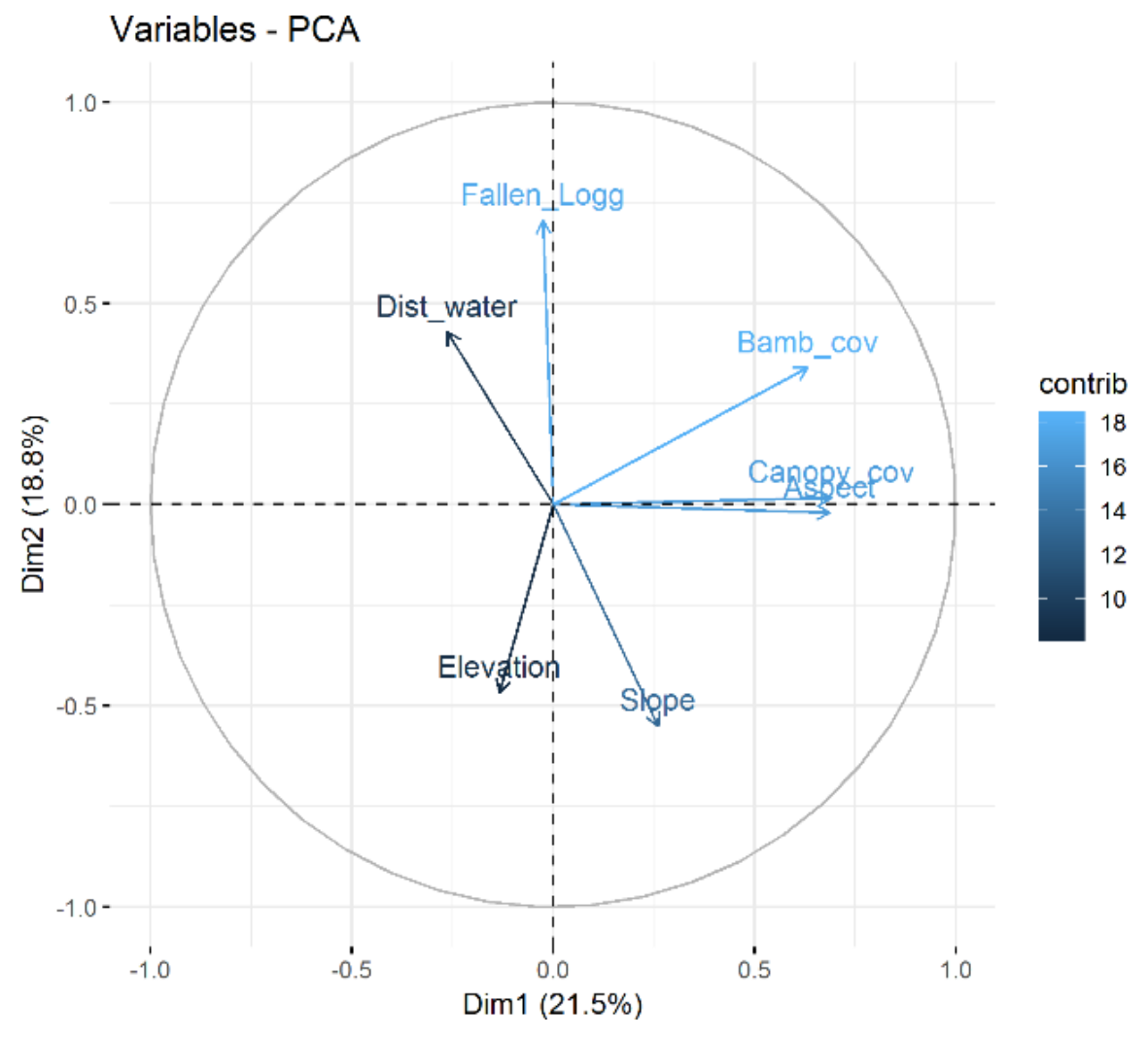




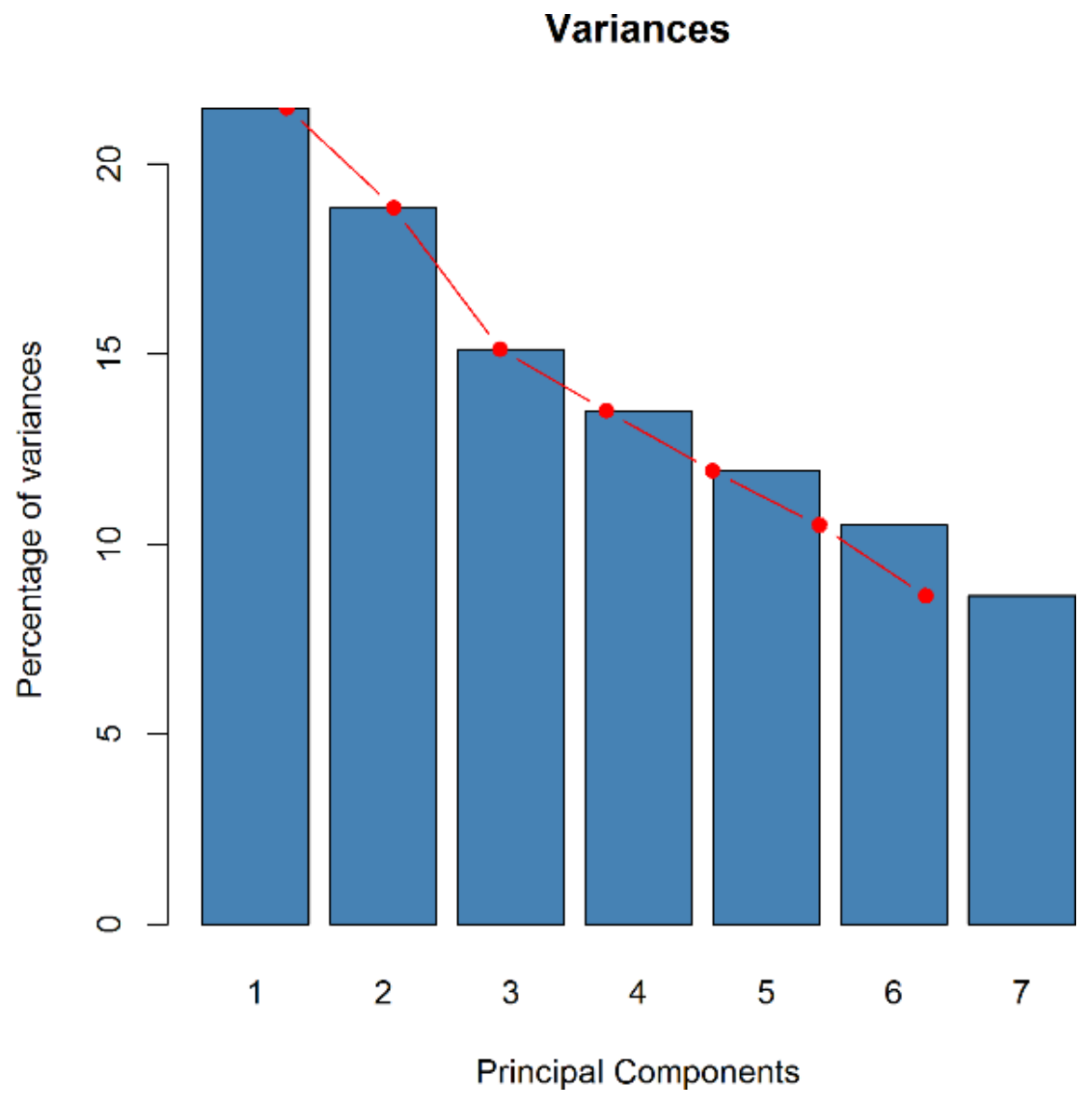

Figure 7Bi-plot of principal component analysis (PCA) showing the relationship of habitat variables(right); a scree plot consisting a graph of the eigenvalues/variances associated with components (left)

\section{Hosted file}

MAPS and FIGURES.docx available at https://authorea.com/users/355878/articles/478970distribution-and-habitat-attributes-associated-with-the-himalayan-red-panda-in-thewesternmost-distribution-range-nepal 